\title{
Germanica
}

\section{Über die "Abenteuerlichkeit des Denkrisikos in der Moderne" - Erinnerungen und Gespräche mit Max Frisch}

"The adventure of daring to think". Memories and conversation with Max Frisch.

"L'aventure du risque de penser »- Souvenirs et entretiens avec Max Frisch

Peter André Bloch

\section{OpenEdition}

Journals

Édition électronique

URL : http://journals.openedition.org/germanica/1223

DOI : 10.4000/germanica. 1223

ISSN : 2107-0784

Éditeur

Université de Lille

Édition imprimée

Date de publication : 1 juillet 2011

Pagination : 183-197

ISBN : 9782913857278

ISSN : 0984-2632

Référence électronique

Peter André Bloch, «Über die "Abenteuerlichkeit des Denkrisikos in der Moderne" - Erinnerungen und Gespräche mit Max Frisch », Germanica [En ligne], 48 | 2011, document 11, mis en ligne le 01 juin 2013, consulté le 06 octobre 2020. URL : http://journals.openedition.org/germanica/1223 ; DOI : https:// doi.org/10.4000/germanica.1223

Ce document a été généré automatiquement le 6 octobre 2020.

(c) Tous droits réservés 


\title{
Über die "Abenteuerlichkeit des Denkrisikos in der Moderne“ - Erinnerungen und Gespräche mit Max Frisch
}

\author{
"The adventure of daring to think". Memories and conversation with Max \\ Frisch.
}

"L'aventure du risque de penser »- Souvenirs et entretiens avec Max Frisch

Peter André Bloch

\section{Begegnungen}

1 Ich erinnere mich an zahlreiche Begegnungen mit Max Frisch ${ }^{1}$ : anlässlich seiner öffentlichen Ansprachen, offiziellen Reden und Stellungnahmen; an Lesungen und Gespräche nach Theateraufführungen im Kreis von Schauspielern und Kritikern; sodann im intimeren Rahmen der freundschaftlichen Treffen der Gruppe Olten, an denen er als tragendes Gründungsmitglied sehr oft teilnahm. Ich traf ihn mehrmals auch - in Berzona, Zürich und Küsnacht - mit Studenten zur wissenschaftlichen Befragung über sein Werk, inbezug auf sein sprachliches Bewusstsein ${ }^{2}$, seine Berufsinteressen als Schriftsteller ${ }^{3}$ und seine politischen Intentionen und Einflussmöglichkeiten, über Zensur und seine Erfahrungen mit Verlagslektoren ${ }^{4}$; Ich bin mit Frisch an den Literaturtagen in Olten und Solothurn in Kontakt gekommen, des öftern in Sils-Maria im Engadin, wo er gerne in den Ferien weilte, bei Spaziergängen oder zu kurzen Gesprächen im Nietzsche-Haus. Die Begegnungen waren vielfältig, Frisch war oft in Begleitung, kannte sich in der Geschichte seiner durchwanderten Landschaften gut aus.

2 Frisch konnte sehr leutselig-offen sein, wenn er sich unter Freunden wähnte oder man ihn kurz in einem Bergrestaurant begrüsste; er verhielt sich eher reserviert- 
zurückhaltend, oft geradezu abweisend, in der Anwesenheit ihm unbekannter Menschen; wirkte überaus hilfsbereit und persönlich engagiert, wenn es um gemeinsame Anliegen ging; reagierte angriffig-kämpferisch, wenn er als "moralische Instanz" wegen Unrecht, Unterdrückung oder erniedrigendem Machtgebaren um Hilfe angegangen wurde. Er konnte sehr launisch auf irgendeine Bemerkung reagieren, den Kontakt unvermittelt abbrechen ${ }^{5}$. Wie jeder Mensch hatte er unterschiedliche Gesichter, je nach Situation und persönlicher Befindlichkeit; was gleich blieb, waren sein Scharfsinn, seine wache Präsenz, seine Beobachtungsgabe. In der Öffentlichkeit hat ihn vor allem sein prinzipielles Misstrauen gegen alle freiheitsmindernden Interessen staatlich-politischer, unternehmerisch-sozialer, religiös-philosophischer, ästhetischer oder auch kulturkritischer Art ausgezeichnet. In dieser Hinsicht wusste er sich identisch mit sich selbst, reagierte unerbittlich, zeigte sich solidarisch mit Geschädigten oder Bedrohten, zum persönlichen Einsatz bereit. Dies hat ihm in weiten Kreisen viel Sympathie, Respekt und Vertrauen eingetragen, unabhängig von seinem unbestrittenen hohen literarischen Rang; auch dann, wenn man anderer Meinung war als er selbst.

\section{Demokratie als Chance}

Wie Dürrenmatt profilierte er sich als Kritiker der sich propagandistisch befehdenden politischen Machtblöcke der Nachkriegszeit, mit einem wachsenden Misstrauen gegenüber Kommunismus und Kapitalismus, deren Ideologien er unentwegt in Frage stellte. Doch während Dürrenmatt diese eher von ihrer absonderlich-absoluten Seite her zu Ende dachte und jedes einseitige Machtdenken durch gegenläufige Prinzipien und grotesk sich steigernde Missverständnisse ad absurdum führte, ging Frisch unerbittlich vom Ernst der Lage aus, denunzierte die Machenschaften der Machtbesitzenden, deren Gewaltbereitschaft in der Beeinflussung der öffentlichen Meinung durch Propaganda und Fehlinformationen, in der Verdrehung von Recht und Gerechtigkeit. Er selbst stammte aus eher einfachen Verhältnissen, achtete daher mit Umsicht auf Möglichkeiten von Erfolg und Einfluss, durchschaute die Mechanismen von Machterwerb und gesellschaftlichem Aufstieg, kannte den Unterschied zwischen persönlicher Identität und Rollenspiel und die daraus entstehenden möglichen Konflikte. Als Schweizer Bürger war er schon in jungen Jahren mit der Politik umsichtig-ängstlicher Kompromissbereitschaft seines Kleinstaates vertraut, mit dem stillschweigenden Akzeptieren der damit zusammenhängenden Konzessionen, im Namen der herrschenden Umstände. Aber nach dem Kriegsende ging er daran, die auf den eigenen Vorteil bedachte Schweizer Abwehrpolitik, die kategorische Überlebensstrategie seines Landes gegenüber Hitler-Deutschland und später wieder gegenüber den Grossmächten $\mathrm{zu}$ untersuchen und in ihrer grundsätzlichen Fragwürdigkeit an den Pranger zu stellen. Er verlangte von sich und den andern klare Positionierungen inbezug auf eine unbedingte Einhaltung der Prinzipien der Menschenrechte auf der Grundlage demokratischer Rechtsstaatlichkeit, forderte das unbedingte Recht auf freie Meinungsäusserung und praktizierte dieses auch in den grossen innenpolitischen Auseinandersetzungen der Schweiz betreffend der politischen Stellung der Gastarbeiter, der Immigranten, der politischen Minderheiten; er weckte den Argwohn, ja Widerstand gegen nationale Scheinmythen, gegen die Aufrüstung Europas, gegen Atomkraft; stand anlässlich der "Zürcher Globus-Krawalle“ 
auf der Seite der Jungen; warb für die Abschaffung der Schweizer Armee, setzte sich persönlich ein für Regimekritiker im In- und Ausland ${ }^{6}$.

\section{Das Ende der Aufklärung?}

4 An den 8. Solothurner Literaturtagen sprach er 1985 enttäuscht vom Ende der "Aufklärung im Kantschen Sinn"; da deren unbedingte Forderung nach "Mut, sich seines Verstandes zu bedienen“, gescheitert sei, habe er selbst aufgehört im Dienste aufklärerisch-ästhetischer Wahrhaftigkeit zu schreiben. Ein letzter "Aufruf zur Hoffnung" bestehe für ihn nur noch im moralischen "Aufruf zum Widerstand"7. Als dann 1989 sogar bekannt wurde, dass in der Schweiz während Jahren, aus Gründen der inneren Sicherheit, eigenen Staatsbürgern (vor allem linksstehenden Politikern, Gewerkschaftern und auch vielen seiner Freunde) nachspioniert worden war und man über sie insgeheim eine Unmenge von Geheimakten angelegt hatte, bezeichnete er diese als Dokumente "der Ignoranz, der Borniertheit, der Provinzialität", nannte die Schweiz einen "verluderten Staat", mit welchem ihn nur noch der Reisepass verbinde, und weigerte sich demonstrativ, an der 700-Jahr-Feier der Eidgenossenschaft teilzunehmen ${ }^{8}$. Es war für ihn unbegreiflich, dass er, der die Schweiz stets öffentlich zwar kritisch, aber rechtsbezogen - unter die Lupe genommen hatte, vom Verfassungsschutz vier Jahrzehnte lang wegen "Kommunismusverdacht" observiert worden war! Dank seiner literarischen Erfolge wusste er sich bis zuletzt in seinen Urteilen unbestechlich und völlig unabhängig; es sei sein höchstes Gut, die "Abenteuerlichkeit des Denkrisikos" gewagt und durchgehalten zu haben, ohne sich an irgendein bestimmtes Machtdenken zu binden. In dieser Auffassung fühlte er sich als politischer Denker nicht zuletzt auch Kierkegaard und Nietzsche zutiefst verbunden', mit dem Unterschied allerdings, dass er an die Grundgesetze eines demokratischen Rechtsstaates glaubte, mit einem garantierten Minderheitenschutz.

\section{Enttäuschungen}

Enttäuschungen über gesellschaftlich-politische Missstände standen am Ende des langen kämpferischen Lebens von Max Frisch, eines Lebens voller Visionen und Entdeckungen, voller Impulse und Anregungen, gekrönt von viel Anerkennung und Freundschaft. Seine eigentlichen Begabungen waren das Zusehen, das Zeugnis-Ablegen, das kritische Hinterfragen, das protestierende Entwickeln von Alternativen. Und er konnte und wollte nicht schweigen. In diesem Sinne war er kein denkerischer Spieler wie Dürrenmatt, sondern ein Erfinder von Gegenentwürfen. In deren Ernsthaftigkeit liegt seine Überzeugungskraft, und in deren künstlerischer Darstellung seine grösste Leistung. Wie kann man die eigenen Unzulänglichkeiten überwinden, wie seine Ängste meistern, wie seine Einsamkeit in Mitmenschlichkeit verwandeln? Er dachte nicht nur über die unzulänglichen politischen Verhältnisse nach, sondern auch über die Beziehung zwischen den Menschen aller Generationen; über das Altern und den Tod, über die Chancen und Grenzen eines jeden Lebens. Er wurde darob von einem sich ständig mehrenden und schliesslich grenzenlos werdenden Pessimismus erfüllt. Er wusste, dass er sich in seinem Leben immer wieder für die eine oder andere Rolle zu entscheiden gehabt hatte, gleichzeitig aber auch, dass er sich dabei immer selber zusah und sich schliesslich als sein eigenes Experiment erfuhr. Er wusste, dass er den Ton 
änderte, wenn er zu den andern - nach aussen - sprach oder wenn er sich schreibend gleichsam mit sich selbst unterhielt. Oder aber - das eine mit dem andern verbindend schreibend gleichzeitig mit mehreren Registern spielte; was zu einem der auffälligsten Kennzeichen seiner Kunst wurde, indem er eine Thematik grundsätzlich auf mehreren - sich entsprechenden oder gegenläufig verlaufenden - Perspektiven zu entwickeln verstand, um die Ambivalenz aller Phänomene in ihrer zeitlichen Veränderbarkeit zu erfassen, auf der Stufe des Sich-Entfaltens, Sich-Vollziehens und aus der Distanz heraus Verstehens. Aus dieser Spiegeltechnik entwickelte sich für ihn die so bezeichnende experimentelle - Lehrhaftigkeit, mit je anderen Aspekten und Vollzügen, Einsichten und Verhaltensmöglichkeiten. In einem Gespräch über den eigenen Darstellungsstil sprach Frisch vom klaren Unterschied zwischen einer öffentlichen Rede, wo er in eigenem Namen Klartext rede, und einem von ihm redigierten literarischen - d.h. mehrdimensionalen - Dramen- oder Erzähltext, wo er versuche, sich in der Rolle einer Figur auszudrücken, d.h. sie nicht nur durch die eigenen Aussagen, sondern auch durch Wortwahl, Sprachtönung und Satzrhythmus zu charakterisieren, im Rahmen der von ihm eingesetzten - übergeordneten - Erzählerinstanz sowie des vorgegebenen Handlungsablaufs ${ }^{10}$.

\section{Satz und Gegensatz}

6 Frischs Werk lebt von Setzungen und ihrem Gegenteil: Als reisender Journalist ist er in seinen schriftstellerischen Anfängen Zeuge, Beobachter, nicht Opfer; daraus gewinnt er die Doppelposition seiner Darstellungsmöglichkeiten: von innen her authentisch zu sein und gleichzeitig aus kritischer Distanz heraus die reflexive Freiheit zu haben, die eigenen Reaktionen $\mathrm{zu}$ fassen und die daraus erwachsenden Konsequenzen weiter $\mathrm{zu}$ spinnen. Alle seine Werke leben in dieser schöpferischen Spannung von These, Antithese und Hypothese: "Ich bin nicht Stiller" - so lautet der als Widerspruch formulierte erste Satz des entsprechenden Romans, mit den sich daraus entwickelnden Folgen: Was wäre, wenn Stiller tatsächlich nicht Stiller wäre? - Am Ende des Romans ist er es aber, wenn auch in veränderter Form: Die Selbstflucht hat ihn zur Fiktion geführt, zur Selbsterfindung in allen möglichen Variationen, was dieses jeweils neue Ich zu neuen Zwängen und $\mathrm{zu}$ wiederum aufgegebenen Alternativen führt, die sich in Gegenbildern aufheben, welche neue Identitätsformen, entsprechende Identitätskrisen und damit immer neue Fragen auslösen: Heisst sich selber sein denn unbedingt glücklich, integriert und schöpferisch sein? Führt Selbstekstase zur Erfüllung oder zum Selbstverlust? Was heisst Beziehungsfähigkeit, was Lebenskunst? - Frischs Figuren stehen immer in der Spannung zwischen mehreren Projektionsmöglichkeiten, die ihre Erscheinung bestimmen und gleichzeitig in Frage stellen ${ }^{11}$. Als Unternehmer erscheint Biedermann vorerst wohl als Ehrenmann, erweist sich später auch als heimlicher Betrüger, ist in diesem Sinne sowohl Opfer als auch Verursacher der Brandstiftung. Auch Gantenbein lebt eine Doppelexistenz: gibt vor, blind zu sein; Homo Faber glaubt, ein freier, ungebundener Reisender zu sein, erweist sich aber als viel mehr in die Wirklichkeit involviert und von seiner eigenen Vergangenheit viel abhängiger als vermutet: Es geht um Verdrängung, um Selbstbetrug, um aufgebaute Illusionen und deren Auflösung. Die Vergangenheit holt das dargestellte Ich oft ein, Erinnerung wird Fakt, und damit vergegenwärtigte oder neue Schuld. Handelt es sich dabei um Projektionen oder um Selbstrechtfertigungen, um erworbene Vorurteile, um sich positiv von andern abzugrenzen? Dies ist in Andorra beim Lehrer so, beim Arzt und 
beim Pfarrer oder auch beim Wirt: sie kommen ihren urtümlichen Verpflichtungen nicht nach, passen sich an, entwerfen von sich in der Öffentlichkeit und vor sich selbst ein Bild, das sich als falsch, als verlogen oder zum Selbstschutz erfunden erweist. Daraus entsteht der erkenntnishafte Leitsatz: "Du sollst Dir kein Bildnis machen“. Selbst Andri möchte sein wie die andern, was er ja eigentlich wäre, wenn nicht zwischen ihm und den andern ein anderes Selbstbewusstsein stünde, das sich bei ihm mehr und mehr verstärkt. Und als er den ganzen Abgrund der Vorurteile und verletzenden "Wahrheiten" erfährt, wird er eins mit seinem andern Ich, aus Verzweiflung und Stolz, aus Einsicht in die Tragik angenommener Wahrnehmungen, die in sich lebensfeindlich, d.h. tödlich, sein können.

\section{Max Frischs Postulat der Vielgesichtigkeit}

7 In den Biographien und Abhandlungen über Max Frisch steht die Suche nach seiner eigenen Persönlichkeit im Vordergrund. Was machte ihn zum Schriftsteller, zum Künstler, zum Erfolgsautor, zum politischen Denker und Moralisten? Steht er in der Nachfolge Nietzsches in der Forderung nach Selbstbestimmung in der Überwindung seiner selbst? Oder erweist sich eben gerade dieses Suchen, über sich hinaus zu gelangen, als Irrweg, als Versuchung, als Selbstherausforderung, als Griff nach den Sternen, um über die vorgezeichneten Grenzen seines Schicksals hinaus zu gelangen? Besteht Frischs Modernität gerade darin, den Menschen als eigenen Selbstentwurf zu verstehen, mit dem damit verbundenen Abenteuer des Scheiterns? Frischs Tochter hat ein persönlich berührendes Dokument ihrer Nicht-Beziehung zu ihrem Vater dargestellt, den sie eigentlich nicht als solchen kennen lernte. Ihre Wünsche blieben unerfüllt, sie fühlte sich allein gelassen, vor allem durch seine Beziehung mit andern Frauen, die sie als Konkurrentin der eigenen Mutter nicht akzeptieren konnte. Immerhin, sie setzte sich zu ihm in Spannung und entwickelte auf diese Weise eine Beziehung auf Distanz ${ }^{12}$. Auch Volker Weidermann versucht in seiner Biographie dem Leben Frischs nachzugehen, indem er die einzelnen Stationen nacherzählt, unter Beziehung vieler Dokumente und auch mit Hilfe verschiedener Gespräche mit Zeitzeugen, mit stetem Bezug zu den damals entstandenen Werken, in der Entwicklung ihrer Thematik mit starkem Bezug auf seine Zeitgenossenschaft ${ }^{13}$. Kenntnisreich und durch ihre nähere Bekanntschaft mit Frisch und seinem Freundeskreis auch dazu ermächtigt, setzt sich Beatrice von Matt mit Frischs Künstlertum auseinander, indem sie ihre persönliche Betroffenheit durch die Person Frischs, sein Werk und seine Wirkung darstellt. Sie versucht auf die Eigenart seiner Selbstsuche einzugehen, auf seine leidenschaftlichen Auseinandersetzungen mit seiner Zeit und deren Herausforderungen an Moral und künstlerischer Verantwortung ${ }^{14}$. Auch Julian Schütts "Biographie eines Aufstiegs", Teil I versucht Frischs Entwicklung in der Spannung zwischen persönlichem Engagement und künstlerischer Verantwortung nachzuzeichnen, zwischen dokumentarischer Verpflichtung, poetisierender Fiktivität und schreibender Lust ${ }^{15}$. Denn es stellt sich bei ihm tatsächlich immer wieder die Frage: Wurde er durch sein angenommenes Leben zum Künstler? oder erfand er sich selbst im Nachhinein als nachdenkendes, schreibendes Ich im sinne einer erklärenden Rechtfertigung seines Selbst durch die Kunst? Wurde er damit zu seiner eigenen Fiktion? als unverstandener Prophet im eigenen Land, im Gespräch mit den literarischen und philosophischen Leitfiguren seiner Zeit, denen er durch sein Temperament und seine hohe darstellerische Kunst, durch sein Spiel mit 
unterschiedlichen Kausaleffekten und provokanten Parabeln unvergessliche Spiegelbilder vorstellte, im Versuch die Tragik seiner Epoche zu verstehen, um das Schlimmste mit seinem Scharfsinn zu verhüten.

\section{Frisch als sein eigener Interpret}

Beim Diskutieren über seine Texte wurde man zum Partner des Autors. Er nahm die Befragung seiner Texte sehr ernst, freute sich über aufgeworfene Verständnisprobleme oder gestalterische Überprüfungen, über Fragen thematischer oder kompositorischer Art. Und dabei wurde manifest, wie bewusst er jeweils beim Konzipieren und Ausführen seines Werks vorgeht, beim Übertragen seiner persönlichen Vorstellungen auf eine sich verfertigende Textwirklichkeit, die er ganz auf sein Lesepublikum hin bezieht, auf dessen Verständnismöglichkeiten, mögliche Assoziationen, Parteilichkeiten oder Vorlieben. Beim Schreiben stellt er sich jeweils seinen Leser vor, wie er selbst spontan zugab, um sich für diese oder eine andere Darstellungsform zu entscheiden, mittels einer Rede, die er persönlich in seinem Namen vortrage, oder in einem bewusst literarisch konzipierten, in sich autonomen Text, den der Leser - als Erzählung oder Roman, als Drama oder Lehrstück - in seiner ästhetisch gefassten, objektivierten Vermittlung übernehme. Mit dieser Kunst der Vermittlung setzte er sich mit grossem Interesse auseinander; wurde im Ergründen dieser - interpretatorisch interessanten "objektivierten" Sprachwirklichkeiten zum virtuosen Selbstbefrager, indem er vorerst die jeweiligen Fragestellungen auf ihre Richtigkeit hin untersuchte, sodann seine Absichten erörterte, die er mit den Eigenschaften der Textrealisierung verglich, die er mit anderen Ausdrucksmöglichkeiten in Beziehung brachte, um ihre Qualitäten zu evaluieren. Gerne sprach er dabei über das eigene Sprach-Instrumentarium: wie er es handhabte und je nachdem so oder so einsetzte, mit spielerischer Lust und kompositorischer Konzentration auf die damit verbundenen Wirkungsmöglichkeiten. Welche Schwierigkeiten er dabei zu überwinden habe, um den zeitgenössischen deutschen, österreichischen, schweizerischen - Leser zu erreichen, ohne Kompromisse, Verkürzungen oder Aufgabe von Differenzierungen. Er kannte seine Werke auch in fremdsprachigen Übersetzungen, die er mit Vergnügen las, um die Realisierung seiner Absichten in ihrer sprachlichen Verfremdung zu überprüfen; freute sich dabei über neue Aspekte in der Konfrontation mit andern Kulturtraditionen, die es zu berücksichtigen gelte. Vor allem ging es ihm schliesslich auch um ein neues Verständnis von schriftstellerischer Arbeit.

\section{Zum Rollenverständnis des Schriftstellers}

Unser zweites Gespräch, in Frischs Zürcher Wohnung, betraf sein Rollenverständnis, d.h. sein "schriftstellerisches Engagement", im Zusammenhang mit den damaligen Schweizer Literaturtagen. Er hatte als Gast den bekannten Schweizer Kommunisten Konrad Farner (1903-1974) eingeladen, Kunsthistoriker und Essayist, der auf Wunsch Helene Weigels am 31. Oktober 1956 im Berliner Ensemble die Trauerrede für Bertolt Brecht gehalten hatte und bei seiner Rückkehr nach Thalwil (Zürich), zusammen mit seiner Familie, massiven Bedrohungen ausgesetzt gewesen war. Frisch hatte sich gegen die organisierten Hasskampagnen gewendet und sich mit Farner solidarisiert, in öffentlicher Verteidigung des Rechts auf Meinungsfreiheit; er unterstützte ihn, wie 
Konrad Farner mir anvertraute, auch finanziell. "Wissen Sie", sagte Frisch zu mir, "es ist einfach, für die Schweizer Werte einzustehen, wenn alle der gleichen Meinung sind. Wenn aber jemand ausschert, indem er Auffassungen vertritt, die man als staatsfeindlich verstehen kann, dann wird es mit der Meinungsfreiheit schwierig, auch in unserem - demokratischen - Land, die wir uns gegen den Kommunismus Stalinistischer Observanz wehren und uns über die Verfolgung Andersdenkender in fremden Ländern aufregen. In dieser Hinsicht ist es vielleicht gerade auch die Aufgabe eines Schriftstellers, wie ich es bin, der ich anerkannterweise wichtige demokratische Standpunkte vertrete, schützend einzugreifen. Aber es ist wirklich nicht einfach: Ich habe so viele böse Briefe erhalten, vor allem auch anonyme, dass man wirklich Angst haben könnte! Ich habe Farner zu unserem Gespräch als Zuhörer eingeladen, und ihm auch erlaubt, einzugreifen, wenn ich etwas sage, was nicht stimmen sollte. Es gibt eben nicht nur ein Engagement für sich, Toleranz gegenüber Andersdenken gehört ebenso dazu wie eine offene Diskussion!“ [Während unserem Gespräch hat Farner, der unter schweren Depression litt, interessiert zugehört, aber nicht eingegriffen; erst später hat er von den öffentlichen Demonstrationen gegen ihn und seine Familie berichtet ${ }^{16}$.

Meine erste Frage betraf Frischs Vorwort zum "Tagebuch 1946-1949", in welchem er die eigene Person in den Hintergrund stellt zugunsten seiner Eigenschaft als Zeitgenosse. "Sie stellen Ihr Werk als Zeitmosaik vor; Sie verstehen also Ihr Engagement vor allem auch als gesellschaftsbezogen?" Frischs Antwort war sehr offen, sehr kritisch, sehr analytisch; man erkennt darin seine Fähigkeit, Gedanken zu entwickeln und zu vertiefen, mit persönlichen Argumenten und allgemein verbindlichen Schlussfolgerungen. Er versucht ideologie-kritisch zu argumentieren im Überdenken des eigenen Standpunkts, den er selbstkritisch überprüft, im Sinne von Kants Aufruf zu einer humanistisch geprägten Aufklärung und Nietzsches Postulat eines Neuen Menschentums im "freien Geiste":

MF: "Ich bin überzeugt, dass es keine Literatur gibt, noch je gegeben hat, die nicht gesellschaftsbedingt ist. Zuerst erhebt sich die Frage, ob diejenigen, welche diese Literatur machen, sich dieser gesellschaftlichen Bedingtheit ihres Denkens und ihres Werkes bewusst sind. Dann wäre, wenn ein solches Bewusstsein vorhanden ist, zu fragen, ob dieses ein affırmatives ist, das heisst im Einverständnis mit der vorhandenen Gesellschaft steht, oder ob es dieser gegenüber kritisch ist, intern system-kritisch, oder ob es über die Gesellschaft hinausweist im Sinn einer Veränderung, Weiterentwicklung der Gesellschaft. Sobald wir heute von Engagement sprechen, denkt man ganz selbstverständlich zuerst immer an das direkt politische Engagement, und ich glaube, dass wir auch jetzt davon sprechen. Wir müssen nur eingangs deutlich sagen: Es gibt natürlich sehr verschiedene Arten von Engagement, zum Beispiel auch religiöse. Man könnte sogar noch allgemeiner sagen: überhaupt niemand schreibt, ohne engagiert zu sein; es fragt sich nur, woran. So könnte jemand, ganz vage ausgesprochen, an die Welt, an die Natur, an die Liebe zum Menschen, an sich selbst usw. engagiert sein. Aber wenn wir nun von Engagement sprechen, meinen wir einschränkend das direkt politische Engagement. Dieses kann affirmativ sein, indem sich der Schriftsteller in der bürgerlichen Gesellschaft seiner gesellschaftlichen Bedingtheit bewusst ist und aus dieser Bewusstheit heraus diese Gesellschaft bejaht, oder kritisch. Die Hauptfrage für uns alle ist natürlich die: Was ist der Effekt des politischen Engagements? Oder schlicht gesagt: Bewirkt es etwas, oder bewirkt es nichts? Dazu müsste ich, nachdem ich schon lange Zeit geschrieben habe, sagen, dass ich schon alle möglichen Standpunkte eingenommen habe, ohne heute bei einem definitiven angelangt zu sein. Gegenüber dem direkt politischen Engagement im Sinne von Agitprop - was nicht unbedingt marxistisches Agitprop sein muss; es gibt bürgerliches Agitprop, religiöses Agitprop usw. - habe ich Zweifel, ob es wirklich etwas bewirkt. Das klassische Beispiel dafür aus jüngster Zeit ist Bertolt Brecht, der ohne Zweifel ein Schriftsteller ersten Ranges war, was man früher einen Dichter genannt hat, der aber auch 
ebenso ohne Zweifel eine ganz bestimmte Tendenz verfolgt hat. Was hat er bewirkt an Bewusstseinsveränderungen in der westlichen Welt, ist die Frage. Denn seine ganze Literatur ist an die westliche Welt adressiert, und wir wissen, dass er heute ein Klassiker ist, als solcher konsumiert wird und im Grunde ohne jede Wirkung bleibt. Er ist ein Bestandteil des bürgerlichen Kulturbetriebes geworden. Aus diesem Beispiel müsste man schliessen, das direkt politische Engagement bewirke überhaupt nichts, selbst dann, wenn es dichterische Qualität erreicht, wie im Fall Brecht; es sei wirkungslos, indem es integriert wird." ${ }^{17}$

11 Ich fragte ihn, ob es nicht vielleicht gerade das Dichterische an Brecht sei, die ästhetische Komponente seines Schreibens, was ihn zur Unwirksamkeit verurteile; indem er das Politische ins Poetische transzendiere und damit ästhetisch mit dem Nimbus des Überzeitlichen, den Zuschauer selbst nicht mehr direkt Betreffenden, entschärfe.

Dazu MF: "Das ist wohl richtig. Es mündet dann ein in den bürgerlichen Literaturbegriff Literatur als etwas Höheres im Sinne von etwas über den Bedingtheiten frei Schwebendem. So werden ja weitgehend auch alle Klassiker (wenigstens die älteren: Goethe, Schiller, Kleist usw.) konsumiert. Die tendenziöse Brisanz von Schiller ist im Allgemeinen, so wie er gelesen und unterrichtet wird, weg. (Es gibt zwar heute Inszenierungen, die sie wieder entdecken.) Der bürgerliche Begriff von Literatur, zum Beispiel vertreten durch Emil Staiger, fasst Literatur als etwas auf, das über den gesellschaftlichen Bedingtheiten schwebt; es ist gewissermassen der ,Weltgeist an sich', der sich hier in ästhetischen Formen ausgiesst." ${ }^{18}$

Ich versuchte Frisch bewusst $\mathrm{zu}$ machen, dass es sich um zwei unterschiedliche Konzeptionen von Literatur handle, die beide nebeneinander ihre Tradition und auch ihre Berechtigung haben: Staiger gehe von einem Dichtertum aus, das sich anschicke, die Welt abzubilden, um sie in ihrem So-Sein, ihrer Schönheit oder ihrer Tragik, wiederzugeben; dazu suche er sich die dazu passenden Idealtypen, die er verstehend interpretiere; Frisch seinerseits denke an ein Dichtertum, das die Welt zu verändern suche; wozu er eine entsprechende Ästhetik und damit auch ein anderes - erweitertes Literaturverständnis entwickle. Vielleicht habe es aber diese emanzipierende Literatur schon früher gegeben, nur habe man sie vielleicht anders gelesen: in der Schönheit der emanzipatorischen Visionen der Lust der Selbstfindung oder der Selbstbefreiung - und weniger im daraus entstehenden Leid oder in der verzweifelten Resignation, im Verlust der Träume oder der Hoffnung? Da würden sich vielleicht Brücken schlagen lassen zwischen den beiden Auffassungen, weil die Übergänge zwischen Schönheit und Hässlichkeit, Erfüllung und Verzicht meist fliessend sind und die eine Situation ohne die andere nicht vorstellbar bleibt. Max Frisch lenkte ein; denn es ging ihm in erster Linie um das Bewusstsein des Schreibenden oder des Interpretierenden gegenüber der jeweiligen Vorstellung des Schreibenden in Bezug auf Gesellschaft und Kunst; d.h. nicht allein um das Abbilden oder Verändern von Wirklichkeit, sondern vielleicht eher um das Schaffen eines neuen Bewusstseins, d.h. um Bewusstseinsveränderung:

MF: "Von dieser ersten versuchsweisen Antwort, vom politischen Engagement aus
weitergehend, bin ich der Meinung (was nachzuweisen wäre), dass es ein Engagement
auch dann gibt, wenn es nicht direkt politisch, wohl aber bewusstseinsverändernd ist, also
einen Umweg macht. Ich habe vorhin auf Brecht zugespitzt von einer erstaunlichen
Wirkungslosigkeit eines brisanten Autors gesprochen. Wenn wir nun sagen, äusserlich
bewirke es (das politische Engagement) gar nichts, dann steht dem gegenüber, dass es in
allen Ländern eine Z e n s u r gibt. Wäre keine Wirkung zu befürchten, gäbe es keine Zensur.
Es gibt hier sicher grosse Unterschiede von Gesellschaft zu Gesellschaft. In einem
faschistischen Land wie Spanien [gemeint ist das damalige ,Franco-Spanien'] sind Dinge
verboten, weil man befürchtet, dass sie ein Bewusstsein herstellen, das der regierenden
Schicht unangenehm wird; das gleiche gilt, wie wir wissen, von der Sowjetunion; es gilt in 
einer sanfteren, verschleierten Weise auch von uns. Also ergibt sich, dass die Herrschenden, die Politiker oder die Machthaber doch etwas befürchten, und dass sie etwas befürchten, muss einen Grund haben; sie müssen gewisse Erfahrungen gemacht haben. Und zwar denke ich nicht nur an die wenigen grossen Fälle, wo eine Dichtung, ein Kunstwerk, etwas Revolutionäres ausgelöst hat - diese Beispiele kennt man, sie sind sehr selten -, sondern einfach daran, dass Dichtung und Literatur die Sprache der herrschenden Schicht immer mit der Realität konfrontiert und damit die Herrschenden verunsichert. Wenn es keine Literatur gäbe, wären gewisse deklamatorische Falschdarstellungen der Wirklichkeit länger möglich. Man könnte immer noch mit dem Begriff ,Vaterland' - der wohl etwas heisst, aber missbraucht wird - den alten Unfug treiben, ebenso mit ,soldatischem Mannesmut' usw. Dadurch, dass die Literatur die Dinge darstellt, wie sie erlebt werden, verunsichert sie die Sprache. Damit wäre ich bei dem, was ich das indirekt politische, bewusstseinsbildende Engagement der Literatur nenne. Ich selber bin der Meinung, um das gleich vorwegzunehmen, dass diese Art Engagement die viel grössere Wirkung hat als das direkte, also das Agitprop. [...] "Die revolutionärste Tat ist von der Literatur her doch wohl immer dort vorgekommen, wo die Literatur die Sprache der herrschenden Schicht zerstört und durch eine andere ersetzt hat. Um es mit einem Beispiel zu sagen: Ein mittlerer, mehr oder weniger naiver Marxist verunsichert mit seiner Literatur die Machtinhaber in unseren Ländern viel weniger als beispielsweise der scheinbar total apolitische Samuel Beckett.."19

13 Auf die Frage, ob er selbst auf eine "neue Sprache“ hinarbeite, welche die traditionell bürgerlichen Sprachkodex verändere, antwortete er in überraschender Offenheit, dass er es mehr und mehr versuche, aber damit sehr spät angefangen habe. "Ich habe sehr lange die von der bürgerlichen Literatur überlieferte Sprache, gepflegt', das heisst, sie übernommen und mit teilweise anderen Meinungen, gefüllt'. Dies sei aber von Werk zu Werk verschieden. ,Objektives Darstellen' und ,gegen etwas Anschreiben' würden sich bei ihm als Pole dauernd beunruhigen, je nach der jeweiligen Absicht oder der situativen Argumentationsweise. Dies sei aber im Grunde bereits bei den alten Griechen so gewesen, auch bei Sophokles oder Aischylos, die man heute vielleicht eher als Bestätiger empfinde, die aber in ihrer Zeit ganz anders gewirkt hätten: als Entwerfer, Utopisten, Projektemacher: "Sie haben eine Welt artikuliert, nicht einfach eine schon artikulierte Welt bestätigt." Es gäbe seiner Meinung nach "kaum ein Land, keinen Weltteil, wo nicht die literarische Existenz per definitionem ,Opposition" bedeute: "Ich gehe noch einen Schritt weiter: Dichten ist in jedem Falle su bve r s $i v$, indem es Dinge neu sieht, nicht definitiv richtig, wobei es von der Erfahrung, von der Erlebensart des Einzelnen ausgeht. Ganz klar ist, wenn ich auf die Heimat anspiele - also den Ort, wo ich aufgewachsen bin, was nicht unbedingt mit ,Vaterland' gleichzusetzen ist -: Es ist ein Unterschied, ob ich dort als Sohn eines Kleinbürgers, als Sohn eines Machtinhabers, als Sohn eines Arbeiters oder als Zugewanderter meine Erfahrungen gemacht habe. In jeder dieser Positionen formuliere ich m e in e Erfahrung, und diese Erfahrung deckt sich nie mit der offiziellen Version, wie es sei, ist also von vornherein subversiv." 20

Die Frage nach Rassismus oder Vorurteilen gegenüber Fremden beruhe auf Angst, oft aber auch auf eigenen Erfahrungen. Damit habe er sich besonders in "Andorra" befasst:

Ich habe aber das Bedürfnis nach einer Selbsterkenntnis, entweder einer personellen oder einer kollektiven, zu wissen, wie solche Dinge entstehen, selbst wenn ich sie nicht verhindern kann. Diese Haltung hat eine gewisse Ähnlichkeit mit derjenigen der Philosophie, die erkennen will, und zwar nicht pragmatisch, indem sie sich nur Fragen stellt, auf die sie eine praktische Antwort weiss, sondern die sich für die Fragen an sich interessiert ${ }^{21}$." Es gehe ihm vor allem um das Erlangen der Freiheit, "anders denken zu können. Man hat mit dem Andersdenken noch sehr wenig Freiheit erobert, aber doch die Möglichkeit, was anders ist, sehen zu können "22."Wenn man direkte Kampfschriften verfasse, mache man die Erfahrung, "dass sie immer nur die 
Einverstandenen erreichen und die andern nicht überzeugen. Es ist meine persönliche Meinung, dass ich mit einem Text, der nicht direkt meine politische Überzeugung verbalisiert, aber eine Darstellung des Konkreten gibt, mehr erreiche (mehr zu erreichen hoffe), indem ich den Partner vorher, verunsichere' und ihn damit frei mache, das Dargestellte neu zu sehen und zwar der Situation angemessener $z u$ sehen. [...] Im Übrigen kommt es natürlich immer sehr auf die Qualität an (ein schwieriger Begriff); jedenfalls sind wir uns wohl darin einig, dass ich vor einem Text, der mir gesinnungsmässig entspricht, nicht einfach umfalle und ihn als Literatur bezeichne. Gesinnung ersetzt Kunst nicht." - Über die Rolle des Schriftstellers in der Gesellschaft angesprochen, deutete er diese wie folgt: "Der Schriftsteller liegt näher beim Forscher, beim Philosophen als etwa (ohne dass ich nun die einzelnen Positionen bewerten möchte) beim aktiven Politiker, beim Geschäftsmann oder auch beim Techniker, dessen Interesse es ist, eine technische Nutzaufgabe zu lösen. Wie ein Forscher geht er auf ein nutzfreies oder jenseits von Nutzen liegendes Abenteuer ein, nur mit anderen Mitteln, indem er die Erkenntnis nicht durch eine philosophische Theorie, sondern durch Darstellung fördert ${ }^{23}$."

16 Auf die Frage, wie er sich informiere, um sich eine eigene Meinung zu bilden, antwortete er, da sei "einmal der wohl überdurchschnittliche Konsum an Massenmedieninformationen. Wichtig ist für mich sodann der Umgang mit Menschen, im privaten Rahmen, im Kreis mit Kollegen, Freunden usw. Seit ich nicht mehr Architekt bin, ist eine gewisse Verengung eingetreten: Mit Arbeitern habe ich nun fast nichts mehr zu tun. Ich könnte sie und ihre Meinungen kennenlernen in Biertischgesprächen, aber nicht mehr durch den natürlichen Funktionszusammenhang, wie man ihn als Architekt früher durch die selbstverständliche Zusammenarbeit gekannt hat. - Dann gibt es für mich die Lektüre. Ich lese weniger literarische Werke als Sachbücher - Feature im Gegensatz zu Fiction - viel anregende, sachliche Information. - Dazu kommt nun für mich vor allem noch das Reisen, der Aufenthalt an andern Orten; ich selber bin nie sehr lange an einem Ort. - Die Hauptinformationsquelle aber, die einem kaum bewusst ist, ist die Erinnerung an früher: an die Kindheit, an die Schule, das Studium, das Militär. Denn sehen Sie: Man weiss dann am meisten über seine Umgebung, wenn man selber drin steckt, nicht als frei schwebender Beobachter, sondern als Träger einer Funktion - als Mitarbeiter, als Mitverantwortlicher ${ }^{24}$."

\section{Versuch einer Bilanz}

17 Wer mit Max Frisch über Fragen von Literatur und Gesellschaft diskutiert hat, weiss, wie logisch-konsequent und wie leidenschaftlich-engagiert er denken und argumentieren konnte. Seine Argumente blieben nie abstrakt, sondern waren stets von anschaulichen Bildern und konkreten Erfahrungen getragen. Als Gesprächspartner blieb man eingebunden in das von ihm entwickelte, in sich komplexe, aber klare, weil immer wirklichkeitsbezogene Gefüge von Denk- und Verhaltensstrukturen. Das Suchen nach den Voraussetzungen des Denkens und Handelns, des Leidens und Schuldigwerdens liess ihn im Literarischen zum Entwerfer von Schicksalen werden, an denen er anhand von eigentlichen Beziehungsgeflechten nach innen und aussen das Wechselspiel von Selbsttätigkeit und Mitvollzug, von aktiver Verantwortlichkeit und passivem Getriebensein, von Vereinsamung und fast selbstverständlicher Mitmacherei darstellte. Damit griff er mit der ihm eigenen Darstellungskraft - in der Nachfolge Nietzsches und auch Brechts - Grundfragen modernen Menschseins auf, die ihn zu einem der grossen Vertreter der deutschsprachigen Nachkriegsliteratur machten - im 
Spannungsfeld zwischen unterschiedlichen Ideologien, technischen Zwängen und persönlichen Glücksansprüchen.

\section{NOTES}

1. Cf. Peter André Bloch, "Max Frisch comme interlocuteur et comme narrateur. Mes Entretiens avec l'auteur de Stiller sur des questions politiques et culturelles et notamment sur la création littéraire“, in: Max Frisch. La Suisse en question ? Éd. Philippe Wellnitz, Strasbourg: Presses universitaires 1997, p. 21-49.

2. Cf. Peter André Bloch und studentische Arbeitsgruppe, Der Schriftsteller und sein Verhältnis zur Sprache, dargestellt am Problem der Tempuswahl. Bern: Francke Verlag 1971. (Max Frisch: p. 68-81).

3. Cf. Peter André Bloch / Edwin Hubacher und studentische Arbeitsgruppe, Der Schriftsteller in seiner Zeit. Schweizer Autoren bestimmen ihre Rolle in der Gesellschaft. Bern: Francke Verlag 1972. (Max Frisch: p. 17-35).

4. Cf. Peter André Bloch / Otto F. Walter und studentische Arbeitsgruppe, Gegenwartsliteratur. Mittel und Bedingungen ihrer Produktion. Bern: Francke Verlag 1975. (Max Frisch: p. 403-404).

5. Als bei einer Befragung eine Gruppe von Studentinnen Max Frisch die Frage stellte, warum im Stiller die weiblichen Figuren nur in der Perspektive von Männern dargestellt seien, verweigerte er die Antwort. Er sei keine Tankstelle für phantasielose Germanistinnen, meinte er; es sei wohl besser, das Verständnis zu einem Werk selbst zu finden, als den Autor darüber zu befragen.

6. Cf. Julian Schütt, Max Frisch - Biographie eines Aufstiegs. Suhrkamp Verlag 2011. / Vgl. dazu auch: MF, "Tagebuch 1946-1949: Autobiographie“, in: Max Frisch, Pro Helvetia Dossier, Reihe Literatur 2, éd. Arthur Zimmermann, p. 118-121.

7. Cf. M.F. Die Schweiz als Heimat? Versuche über 50 Jahre. Éd. Walter Obschlager, Frankfurt a.M. 1990.

8. Cf. DU. Die Zeitschrift der Kultur, Heft 12, Dezember 1991, Max Frisch 1911-1991, p. 103 ; dazu auch Marco Solari, Nein, ich halte die Schweiz nicht für verludert, p. 109.

9. Cf. Peter André Bloch, «La duplicité du regard: voir et être vu. Nietzsche et ses conséquences: Exemples: Max Frisch et Friedrich Dürrenmatt», in: Voir \& Être vu. Réflexions sur le champ scopique dans la littérature et la culture européennes. Études réunies et présentées par Peter Schnyder et Frédérique Toudoire-Surlapierre, Éditions L'improviste, 2011, p. 377-390.

10. Cf. Peter André Bloch / Edwin Hubacher und studentische Arbeisgruppe, Der Schriftsteller und sein Verhältnis zur Sprache. Schweizer Autoren bestimmen ihre Rolle in der Gesellschaft. Berne: Francke Verlag 1975, p. 69-78.

11. Cf. Gerda Zeltner-Neukomm, "Das Ich ohne Gewähr“, in: Max Frisch, Pro Helvetia Dossier, Reihe Literatur 2, éd. Arthur Zimmermann, p. 99-116 / P.A. Bloch, "Max Frisch: 'Stiller' oder: die formgebende Kraft des Themas"; dans: Festschrift / Mélanges Claude David, Berne: Peter Lang 1986, p. 129-144. (Version complétée dans Stiller. La quête d'identité dans le roman moderne. Actes du Colloque Max Frisch. Éd. Philippe Wellnitz, Strasbourg: Presses universitaires 1996, p. 7-30). 
12. Cf. Ursula Priess, Sturz durch alle Spiegel. Zürich: Ammann-Verlag 2009.

13. Volker Weidermann, Max Frisch. Sein Leben, seine Bücher. Köln: Kiepenheuer \& Witsch 2010.

14. Beatrice von Matt, Mein Name ist Frisch. Begegnungen mit dem Autor und seinem Werk. München : Nagel \& Kimche 2011.

15. Julian Schütt, Max Frisch - Biographie eines Aufstiegs. Teil I, Berlin: Suhrkamp Verlag 2011.

16. Notes privées $P A B$.

17. Cf. Peter André Bloch/Edwin Hubacher und studentische Arbeitsgruppe, Der Schriftsteller in unserer Zeit. Schweizer Autoren bestimmen ihre Rolle in der Gesellschaft. Berne: Francke Verlag 1972, p. 17-18.

18. Ibid., p. 18.

19. Ibid., p. 19-20.

20. Ibid., p. 21.

21. Ibid., p. 21-22.

22. Ibid., p. 23.

23. Ibid., p. 23-33.

24. Ibid., p. 35.

\section{RÉSUMÉS}

Cette contribution comporte trois parties: la première est consacrée à Max Frisch dans sa disposition naturelle à communiquer avec son public pour défendre la liberté de penser et d'agir contre toute idéologie et tout pouvoir absolu. Il se considérait à cet égard comme héritier de Nietzsche, fondant sa réflexion sur les nouvelles libertés démocratiques acquises au xxe siècle. Dans la deuxième partie nous évoquerons ses déceptions face à la Guerre Froide, à la manière dont la Suisse de l'après-guerre trahit ses responsabilités morales en tant que pays démocratique et neutre. Dans la structure de ses œuvres, les problèmes d'identité jouent un rôle primordial ; ils reflètent une faille décisive entre thèse et antithèse, conformisme et résistance, entre un sens prononcé de la réalité et une forte disposition pour l'imaginaire et toute vision innovatrice. Dans la troisième partie, nous étudierons Frisch en tant qu'interprète de sa propre œuvre : à quels moyens linguistiques recourt-il dans ses oeuvres, comment caractérise-t-il ses personnages et de quelle manière imagine-il son lecteur idéal ? Frisch plaidait pour une nouvelle conception du travail d'écrivain s'engageant pour un dynamisme culturel ayant pour vocation de changer la conscience de la société et de promouvoir une réflexion indépendante et humaniste destinée à dépasser tout préjugé vis-à-vis des minorités et des différences qu'elles incarnent.

This analysis of Max Frisch's work concerns three aspects of his art. The first part describes him in conversation with his public in order to defend liberty of thought and action against the ideology and will of absolute power. In this respect, he always placed himself in the tradition of Nietzsche, although inspired by the new democratic traditions of the 20th century. The second part deals with his disillusionment with European politics after the Second World War, and his protest against the Swiss tendency to betray moral responsibilities as a democratic neutral country. The structure of his literary work is marked by the existential problem of identity, accompanied by the gap between thesis and antithesis, conformism and resistance, between a 
profound sense of realism and an opposite tendency of imaginative visions and innovation. The third and final part of this analysis thus concerns Frisch's own understanding of himself. He explains how he composes his texts, how he characterizes his protagonists, and how he imagines his ideal reader, including the social function of his art. He stood for a new way of conceptualizing the work of the writer, a notion which involves the adoption of a dynamic cultural stance and an attempt to change public consciousness, at the same time as conducting an independent and humanist reflection about how to overcome the many prejudices against those who do not think like the majority.

Der Aufsatz besteht aus drei Teilen. Am Anfang steht die Beschreibung von Max Frisch als Gesprächspartner, in seiner Bereitschaft zu kommunizieren und sich für die Freiheit des Denkens gegenüber jedem ideologischen Missbrauch einzusetzen. Er versteht sich in der Nachfolge Nietzsches, auf der Grundlage jedoch der vor allem im 20. Jahrhundert errungenen demokratischen Freiheiten. Der zweite Teil betrifft vor allem Frischs Enttäuschungen: seinen Ärger über den Kalten Krieg, über den Umgang der Schweiz mit ihrer Verantwortung als neutrales Land sowie über die staatlichen Übergriffe auf die demokratischen Volksrechte. In seiner Persönlichkeit wie in seinem Werk spielt das Problem des Identitätsbruchs eine vorrangige Rolle: Satz und Gegensatz, Anpassung und Widerstand, Wirklichkeitsbezogenheit und visionäre Entwurfsbereitschaft. Im dritten Teil zeigt sich Frisch als sein eigener Interpret: mit welchen künstlerischen Mitteln er seine Werke komponiert, seine Figuren sprachlich zu charakterisieren sucht; wie er sich seinen Leser vorstellt, welche gesellschaftlichen Funktionen er seinem Werk beimisst. Frisch plädierte für ein neues Verständnis schriftstellerischer Arbeit, im Hinterfragen von Sprachmanipulationen im Dienste von Macht und Ideologie. Er setzte sich für eine Kultur permanenter Bewusstseinsveränderung ein, für ein unabhängiges philosophisches Denken, zum Überwinden von politischen und sozialen Vorurteilen gegenüber Minderheiten und Andersdenkenden.

\section{AUTEUR}

\section{PETER ANDRÉ BLOCH}

Universität Mulhouse, ILLE 